\title{
Massive abdominal and pelvic myxoma in Carney's syndrome
}

\author{
W G McCluggage, M Y Walsh, C M Thornton, H R McClelland, S T Irwin
}

\begin{abstract}
This report describes a massive abdominal and pelvic myxoma in a patient with Carney's syndrome. A 38 year old woman presented with abdominal distension and a palpable mass, and at operation a large pelvic and abdominal tumour was identified and resected. The surgical specimen consisted of a lobulated mass, which on cut section had a uniform gelatinous consistency. The mass surrounded both ovaries, the appendix, and the upper part of the uterus, but macroscopically did not appear to involve these organs. Histological examination showed plump stellate and spindle shaped cells set in an abundant myxoid stroma, in keeping with a myxoma. Immunohistochemical staining revealed positivity of tumour cells for vimentin, but no reactivity to desmin, $\alpha$-smooth muscle actin, S-100 protein, CD34, or AE1/AE3. This is the first documented case of massive adominal and pelvic myxoma in a patient with Carney's syndrome. Clinicians and pathologists should be aware that myxomas in Carney's syndrome can rarely involve unusual sites other than the skin and heart. (F Clin Pathol 2000;53:558-560)
\end{abstract}

Keywords: abdomen; pelvis; myxoma; Carney's syndrome; immunohistochemistry

Department of Pathology, Royal Group of Hospitals Trust, Grosvenor Road, Belfast BT12 6BL, Northern Ireland

W G McCluggage

M Y Walsh

C M Thornton

Department of Colorectal Surgery, Royal Group of Hospitals Trust, Belfast BT12 6BL, Northern Ireland $\mathrm{S}$ T Irwin

Department of Obstetrics and Gynaecology, Belfast City Hospital, Belfast BT9 7AB, Northern Ireland

H R McClelland

Correspondence to: Dr McCluggage

Accepted for publication 20 October 1999
Carney's syndrome or complex is one name given to a complex consisting of myxomas, spotty pigmentation, and endocrine overactivity. This peculiar syndrome is also known by a variety of names including Swiss syndrome, NAME syndrome, and LAMB syndrome. ${ }^{1}$ Both male and female individuals can be affected and, based on a familial study, Carney et al suggested that an autosomal dominant mode of inheritance was likely. ${ }^{2}$ The syndrome is characterised by a variety of disorders, not all of which are necessarily present in an affected individual. Lesions found in this syndrome include cutaneous and cardiac myxoma, myxoid fibroadenoma of the breast, spotty cutaneous pigmentation, cutaneous blue naevus, pigmented nodular adrenocortical disease, pituitary growth hormone secreting tumour, psammomatous melanotic Schwannoma, and large cell calcifying Sertoli cell tumour of the testis. $^{23}$

Here, we describe a patient with Carney's syndrome, and with a history of cutaneous and cardiac myxomas, who developed a massive tumour involving the abdomen and pelvis, which on histological examination comprised a myxoma. As far as we are aware, this is the first documented report of a massive abdominal and pelvic myxoma in Carney's syndrome.

\section{Case report}

A 38 year old woman, who was known to have Carney's syndrome, presented with a four week history of abdominal distension and a palpable abdominal mass. She had lost four stone in weight over the past two years, most of this in the last six months. On examination she had the typical multiple pigmented skin lesions of Carney's syndrome. These were particularly prominent on the face and the vulval region. Examination of the abdomen revealed a large suprapubic mass arising out of the pelvis and extending to the right iliac fossa. An enhanced computed tomography scan of the abdomen showed ascites and a large irregular mass lesion within the pelvis, the cranial extent of which reached the aortic bifurcation. There was no evidence of metastatic disease. Magnetic resonance imaging confirmed the presence of a large pelvic mass, which appeared intimately associated with the uterus and adnexae.

At surgery, there was two litres of blood stained ascites. A large mass arose from the pelvis and surrounded the superior aspect of the uterus and the right and left ovaries. The mass could not be separated from the left ovary and was adherent to the appendix. Complete excision of the mass was performed together with total abdominal hysterectomy, bilateral salpingo-oophorectomy, and removal of the appendix. The postoperative course was uneventful.

The diagnosis of Carney's syndrome had been made 12 years previously after the development of multiple cutaneous myxomas. She also had surgery to remove a left atrial myxoma. She has three children, two of whom are affected by Carney's syndrome.

\section{Materials and methods}

The surgical specimen was fixed in formalin and sections for histological examination were routinely processed in paraffin wax and stained with haematoxylin and eosin. Immunohistochemistry was performed using a standard streptavidin-biotin peroxidase method (Dako, Copenhagen, Denmark). Sections were stained with monoclonal antibodies to the following molecules: vimentin (Dako), desmin (Dako), $\alpha$-smooth muscle actin (Sigma, Poole, UK), S-100 protein (Diagnostic Products Ltd, Abingdon, UK), CD34 (Serotec, Oxford, UK), and AE1/AE3 (ICN-Flow, Aurora, Ohio, USA). Immunostaining was performed using appropriate positive and negative controls. 


\section{Pathological findings}

The surgical specimen consisted of a $7 \mathrm{~cm}$ length uterus and cervix. A large lobulated tumour measuring $20 \mathrm{~cm}$ in maximum dimension was adherent to the external surface of the superior aspect of the uterus. Sectioning revealed this to have a uniform gelatinous consistency. Several areas of haemorrhage were present. The tumour did not infiltrate the uterus and two grossly unremarkable ovaries and fallopian tubes were identified embedded within the mass. A macroscopically unremarkable appendix was also identified within the tumour.

Histological examination of multiple sections from the tumour showed a uniform appearance throughout. Stellate and spindle shaped cells were embedded within an abundant myxoid stroma (fig 1A). These had plump vesicular nuclei, sometimes with prominent nucleoli, and surrounding eosinophilic cytoplasm. Occasional multinucleate cells were present (fig 1B) but there was no pronounced nuclear pleomorphism. Mitotic figures were not identified. Thick walled blood vessels were present within the tumour but there was no arborising vascular pattern. No lipoblasts or chondroid areas were identified. There were areas of fresh and old haemorrhage with

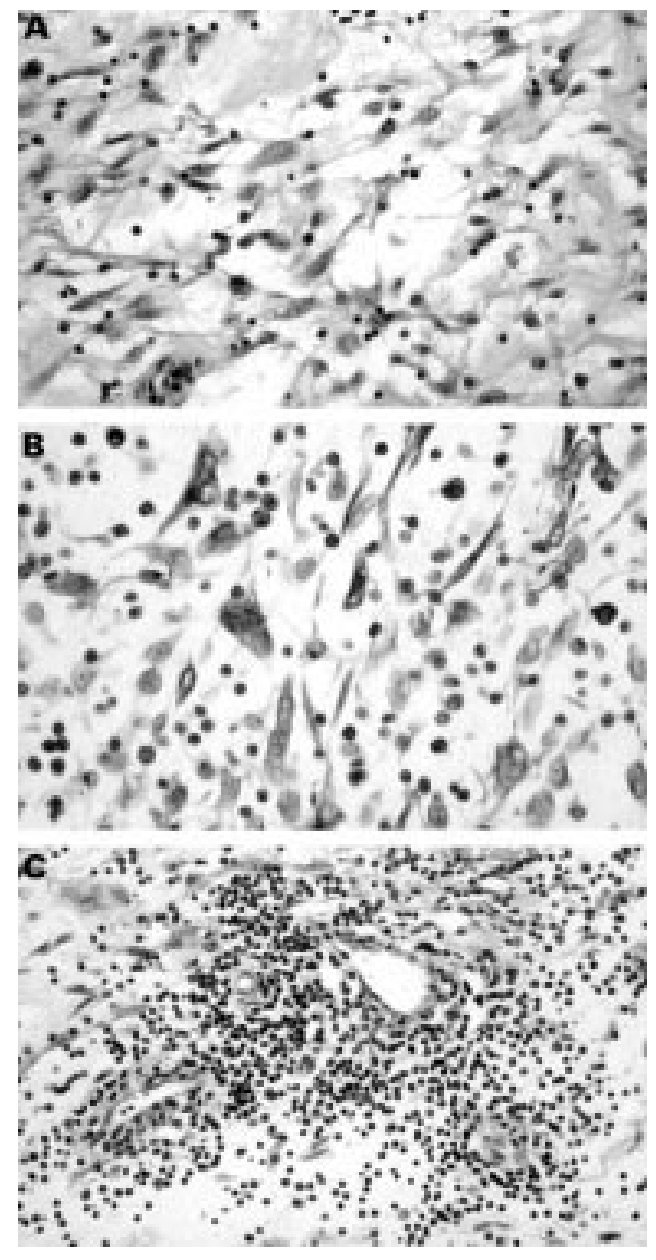

Figure 1 (A) Stellate and spindle shaped cells embedded in an abundant myxoid stroma. (B) Multinucleate tumour cells can be seen in some areas. (C) In some areas there is an intense infiltrate of small lymphoid cells. haemosiderin pigment but no necrosis. There was also a scattered lymphoid infiltrate, which in areas was quite intense (fig 1C). This lymphoid infiltrate was composed of lymphocytes and plasma cells. The tumour extended to the margins of the specimen.

Immunohistochemical staining showed the stellate and spindle shaped tumour cells to be positive with antivimentin but not with the other antibodies. Staining for desmin, $\alpha$-smooth muscle actin, and CD34 highlighted the vascular channels within the neoplasm.

No important microscopic abnormality was identified within the uterus or cervix. The right ovary was histologically unremarkable and the cortical surface of the left ovary was superficially infiltrated by tumour. The mucosa, submucosa, and muscularis propria of the appendix were unremarkable but tumour was seen to involve the serosal surface.

\section{Discussion}

Patients affected with Carney's syndrome often develop myxomas, the most common locations being the skin and the heart. Myxoid change can also occur in mammary fibroadenomas. ${ }^{4}$ Our patient had a history of cutaneous and cardiac myxomas. We are unaware of any previous reports in the literature of an abdominal or pelvic myxoma in Carney's syndrome, nor are we aware of a myxoma of such a large size. It is likely that the lesion attained such a size because of its location in the abdomen and pelvis. In this relatively inaccessible site, the tumour is likely to have grown without noticeable symptomatology. Degenerative changes were present in the form of thick walled blood vessels, an inflammatory infiltrate, and areas of old and recent haemorrhage. These degenerative changes suggest that the lesion was long standing.

The likely behaviour of the neoplasm is not certain. Cutaneous and cardiac myxomas in Carney's syndrome are invariably benign, although cardiac myxomas can be life threatening because of their location. In our patient, although the morphological features were in keeping with a benign myxoma, the large size of the tumour, together with the fact that it was present at the excision margins, suggest that recurrence is a possibility.

At laparotomy it was thought that the neoplasm had arisen from the left ovary. Male patients affected by Carney's syndrome can develop testicular sex cord stromal tumours, especially large cell calcifying Sertoli cell tumours, ${ }^{5}$ and this was thought a likely diagnosis. However, gross and microscopic examination of the resected mass showed that it surrounded both ovaries and only superficially invaded the left ovary. There have been occasional reports of primary ovarian myxomas (not in Carney's syndrome), ${ }^{67}$ but we feel the involvement of the left ovary in our patient was the result of direct spread from the adjacent mass.

The histological appearances we describe are entirely in keeping with a diagnosis of myxoma. Other lesions considered in the differential diagnosis included myxoid smooth muscle 
tumour, myxoid peripheral nerve sheath tumour, myxoid malignant fibrous histiocytoma, myxoid liposarcoma, myxoid chondrosarcoma, gastrointestinal stromal tumour, and aggressive angiomyxoma of pelvic parts. Myxoid smooth muscle tumour and myxoid peripheral nerve sheath tumour were excluded by immunohistochemistry, which revealed no staining with smooth muscle or neural markers. Similarly, a gastrointestinal stromal tumour was thought unlikely because there was no staining of tumour cells with anti-CD34, which is almost invariably positive in this neoplasm. A myxoid malignant fibrous histiocytoma was thought unlikely because tumour cells were bland, without mitotic activity, and because there was no storiform growth pattern. Myxoid liposarcoma was excluded owing to an absence of lipoblasts and because of the characteristic plexiform or arborising vascular pattern. A myxoid chondrosarcoma was excluded because the tumour cells in this are usually plump with an epithelioid appearance. Aggressive angiomyxoma of pelvic parts was also considered but this was thought unlikely because in general this lesion primarily involves the vulva and perineum. ${ }^{8}$

In summary, we describe a massive abdominal and pelvic myxoma in a patient with Carney's syndrome. Although cutaneous and cardiac myxomas are well described in this syndrome, this is the first report of a massive abdominal and pelvic tumour. The neoplasm is likely to have attained such a large size because of its relatively inaccessible location within the abdomen and pelvis. Myxomas arising sporadically in patients without Carney's syndrome are most commonly intramuscular or juxtaarticular in location, ${ }^{10}{ }^{11}$ and the abdomen and pelvis are extremely rare sites. This illustrates that myxomas in Carney's syndrome can rarely develop in sites other than the skin and heart. A myxoma arising in an unusual site might be the first presentation in Carney's syndrome, and clinicians and pathologists should be aware of this and take appropriate steps to exclude the syndrome.

Note in proof: since the writing of this report, the patient has had a massive recurrence of abdominal myxoma (confirmed histologically)

1 Carney JA, Gordon H, Carpenter PC, et al. The complex of myxomas, spotty pigmentation and endocrine overactivity. Medicine 1985:64:270-83.

2 Carney JA, Hruska LS, Beauchamp GD, et al. Dominant inheritance of the complex of myxomas, spotty pigmentation, and endocrine overactivity. Mayo Clin Proc 1986:61: 165-72.

3 Handley J, Carson D, Sloan JM, et al. Multiple lentigenes. Myxoid tumours and endocrine overactivity; four cases of Carney's complex. Br f Dermatol 1992:126:367-71.

4 Carney JA, Toorkey BC. Myxoid fibroadenomas and allied conditions (myxomatosis) of the breast: a heritable disorder with special associations including cardiac and cutaneous with special associations including cardiac an

5 Proppe KH, Scully RE. Large-cell calcifying Sertoli cell Proppe KH, Scully RE. Large-cell calcifying Sertoli
tumor of the testis. Am f Clin Pathol 1980:74:607-19.

6 Costa MJ, Morris R, De Rose PB, et al. Histologic and immunohistochemical evidence for considering ovarian myxoma as a variant of the thecoma-fibroma group of ovarian stromal tumors. Arch Pathol Lab Med 1993:117: 802-8.

7 Tetu B, Bonefant JL. Ovarian myxoma. A study of two cases with long-term follow up. Am f Clin Pathol 1991:95:340-6.

8 Fetsch JF, Laskin WB, Lefkowitz $\mathrm{M}$, et al. Aggressive angiomyxoma: a clinicopathologic study of 29 female patients. Cancer 1996:78:79-90.

9 Steeper T, Rosai J. Aggressive angiomyxoma of the female pelvis and perineum. Am F Surg Pathol 1983:7:463-76.

10 Enzinger FM. Intramuscular myxoma. A review and follow-up study of 34 cases. Am F Clin Pathol 1965:43:10413.

11 Meis JM, Enzinger FM. Juxta-articular myxoma. A clinical and pathologic study of 65 cases. Hum Pathol 1992:23:639 